\title{
Pairing, off-diagonal long-range order, and quantum phase transition in strongly attracting ultracold Bose gas mixtures in tight waveguides
}

\author{
M. D. Girardeau* \\ College of Optical Sciences, University of Arizona, Tucson, AZ 85721, USA
}

(Dated: November 20, 2018)

\begin{abstract}
A model of two 1D ideal Bose gases $\mathrm{A}$ and $\mathrm{B}$ with strong $\mathrm{AB}$ attractions induced by a p-wave $\mathrm{AB}$ Feshbach is studied. The model is solved exactly by a Bose-Bose duality mapping, and it is shown that there is no A-component or B-component Bose-Einstein condensation and no AB-pair offdiagonal long-range order (ODLRO), but both AA-pair and BB-pair ODLRO. After generalization by adding even-wave $\mathrm{AA}$ and $\mathrm{BB}$ repulsions and reducing the strength of the odd-wave $\mathrm{AB}$ attraction by Feshbach resonance detuning, a quantum phase transition occurs between a phase with $\mathrm{AB}$ contact nodes and one with no such nodes.
\end{abstract}

PACS numbers: 03.75.Mn,67.85.-d

Strong interatomic interactions and correlations occur in ultracold gases confined in de Broglie waveguides with transverse trapping so tight that the atomic dynamics is essentially one-dimensional (1D) [1], with confinementinduced resonances [1, 2] allowing Feshbach resonance tuning 3] of the effective 1D interactions to very large values. This has led to experimental verification [4, 5, 6] of the fermionization of bosonic ultracold vapors in such geometries predicted by the Fermi-Bose (FB) mapping method [7], an exact mapping of a 1D gas of bosons with point hard core repulsions, the "Tonks-Girardeau" (TG) gas, to an ideal spin-aligned Fermi gas. The "fermionic Tonks-Girardeau" (FTG) gas 8, 9], a 1D spin-aligned Fermi gas with very strong attractive interactions, can be realized by a 3D p-wave Feshbach resonance as, e.g., in ultracold ${ }^{40} \mathrm{~K}$ vapor [10]. It has been pointed out [2, 8, 9, 11] that the FB mapping [7] can be exploited to map the FTG gas to the ideal Bose gas. The very strong fermion-fermion attraction in the FTG gas leads to fermion pairing and superconductive off-diagonal longrange order (ODLRO) of the two-fermion density matrix [12].

There are a number of models of strongly interacting 1D ultracold gas mixtures which are exactly soluble by generalizations of the FB mapping [13]. In [13, 14] the properties of one such soluble model, a mixture of a TG Bose gas A and an ideal Fermi gas B, with point hardcore $\mathrm{AB}$ interactions, were investigated in detail. Here I shall discuss another model, a mixture of two different ideal Bose gases $\mathrm{A}$ and $\mathrm{B}$, with an $\mathrm{AB}$ interaction of FTG form. This model is exactly soluble, and it will be shown that it has very unusual behavior: The strong $\mathrm{AB}$ attraction destroys the ground state Bose-Einstein condensation (BEC) and single-particle off-diagonal longrange order (ODLRO) of both components $\mathrm{A}$ and $\mathrm{B}$, and it induces both $\mathrm{AA}$ and $\mathrm{BB}$ pairing manifested in superconductive ODLRO of both the two-A and two-B density matrices, although there are no $\mathrm{AA}$ or $\mathrm{BB}$ interactions. Furthermore, there is no AB pair ODLRO in spite of the strong $\mathrm{AB}$ attractions. It will also be shown that if the
$\mathrm{AB}$ attraction is a finite odd-wave attraction rather than the infinite FTG limit, and there is also a repulsive evenwave $A B$ interaction of Lieb-Liniger (LL) delta function form [15], then there is a quantum phase transition as the coupling constants are varied, between a phase in which there are no $\mathrm{AB}$ contact nodes and only the repulsive LL interaction acts, and another phase in which there are $\mathrm{AB}$ contact nodes and only the attractive FTG-like interaction acts.

FTG interaction and nodal structure: The FTG gas is a spin-aligned 1D Fermi gas with infinitely strongly attractive zero-range odd-wave interaction induced by a p-wave Feshbach resonance. It is the infinite 1D scattering length limit $a_{1 D} \rightarrow-\infty$ of a $1 \mathrm{D}$ Fermi gas with zero-range attractive interactions leading to a $1 \mathrm{D}$ scattering length defined in terms of the ratio of the derivative $\Psi^{\prime}$ of the wave function to its value at contact: $\Psi\left(x_{j k}=\right.$ $0+)=-\Psi\left(x_{j k}=0-\right)=-a_{1 D} \Psi^{\prime}\left(x_{j k}=0 \pm\right)$ where the prime denotes the derivative with respect to $x_{j k}[2,8,9]$. The FTG limit $a_{1 D} \rightarrow-\infty$ corresponds to a $1 \mathrm{D}$ zeroenergy odd-wave scattering resonance reachable by Feshbach resonance tuning to a $1 \mathrm{D}$ odd-wave confinementinduced resonance [1, 2, 3]. There are several different zero-range pseudopotentials which generate this contact condition. One representation [8] is $\hat{v}_{o}=g_{o} \delta^{\prime}\left(x_{j k}\right) \hat{\partial}_{ \pm}$ where $\hat{\partial}_{ \pm} \Psi\left(x_{j k}\right)=(1 / 2)\left[\Psi^{\prime}(0+)+\Psi^{\prime}(0-)\right]$. The FTG limit $a_{1 D} \rightarrow-\infty$ is equivalent to $g_{o} \rightarrow+\infty$. This representation explicitly exhibits an odd-wave projection property of the interaction, i.e., it vanishes on even functions of $x_{j k}$.

Since there is no particular symmetry under exchange of particles of the different species $\mathrm{A}$ and $\mathrm{B}, \mathrm{AB}$ scattering in all partial waves is possible, but usually s-wave scattering dominates. However, in the neighborhood of a $\mathrm{p}$-wave $\mathrm{AB}$ resonance, $\mathrm{p}$-wave scattering dominates, and gives rise in $1 \mathrm{D}$ to an odd-wave effective interaction of FTG form [2, 8, 9]. For this it is important to realize that the odd-wave projection property of $\hat{v}_{o}$ requires only local antisymmetry, i.e., it guarantees that if $x_{i}$ is an A-particle position and $y_{j}$ a B-particle position, then 
$\hat{v}_{o} \Psi\left(x_{i}, y_{j}\right)$ is nonzero only if $\Psi$ has a node at $x_{i}=y_{j}$, where $\Psi\left(x_{i}, y_{j}\right)=-\Psi\left(y_{j}, x_{i}\right)$ in an infinitesimal neighborhood of the node. It is not necessary that there be global antisymmetry under such exchange, and indeed, the ground state $\Psi_{0}$ derived in the following sections has no such global antisymmetry. In the presence of both a $1 \mathrm{D}$ even-wave $\mathrm{AB}$ Lieb-Liniger interaction $g_{e} \delta\left(x_{i}-y_{j}\right)$ [15] generated by $3 \mathrm{D}$ s-wave $\mathrm{AB}$ scattering and a $1 \mathrm{D}$ oddwave resonance generated by a $3 \mathrm{D}$ p-wave Feshbach resonance for $\mathrm{AB}$ scattering, the wave function can lower its energy by developing nodes at $x_{i}=y_{j}$ so as to kill the repulsive even-wave interaction and activate the strong 1D odd-wave attraction.

The contact discontinuities of $\Psi$ [1] can also be understood as a zero-range limit $x_{0} \rightarrow 0+$ and $V_{0} \rightarrow \infty$ of the two-body scattering solution for a square well of width $2 x_{0}$ and depth $V_{0}$, where the limit is carried out such that $V_{0} x_{0}^{2}$ approaches a finite, nonzero limit [8, 9]. In the untrapped case, the exterior solution for scattering length $a_{A B} \rightarrow-\infty$ is constant $(+1$ for $x-y>0$ and -1 for $x-y<0$ ), and the interior solution is $\sin [\kappa(x-y)]$ with $\kappa=\sqrt{2 \mu V_{0} / \hbar^{2}}=\pi / 2 x_{0}$ and $\mu$ the effective mass $m_{A} m_{B}\left(m_{A}+m_{B}\right)$; hence $\kappa x_{0}=\pi / 2$ and $V_{0} x_{0}^{2}=(\pi \hbar)^{2} / 8 \mu$. In the zero-range limit the interior kinetic energy $\rightarrow+\infty$ and potential energy $\rightarrow-\infty$, but their sum remains zero, the ground state energy. Since the FTG interaction acts only on odd waves, the FTG interaction $\hat{v}_{0}$ should be written as $\hat{v}_{0}=v \hat{P}_{0}$ where $v$ is the above square well and $\hat{P}_{0}$ is the odd-wave projector.

Many-body ground state: Assuming trapping on a ring, the Hamiltonian consists only of the kinetic energy operators of components A and B plus the AB FTG interaction:

$$
\hat{H}=\sum_{i=1}^{N_{A}} \frac{-\hbar^{2}}{2 m_{A}} \frac{\partial^{2}}{\partial x_{i}^{2}}+\sum_{i=1}^{N_{B}} \frac{-\hbar^{2}}{2 m_{B}} \frac{\partial^{2}}{\partial y_{i}^{2}}+\sum_{i=1}^{N_{A}} \sum_{j=1}^{N_{B}} \hat{v}_{o}\left(x_{i}-y_{j}\right)
$$

where $\hat{v}_{o}$ is the previously defined odd-wave FTG interaction. The wave functions satisfy periodic boundary conditions with periodicity length $L$ (the ring circumference) with respect to all the $x_{i}$ and $y_{i}$. The scattering length $a_{A B}$ is $-\infty$ in the FTG limit. For square well width $2 x_{0}$ nonzero but very small, the unnormalized ground state $\Psi_{0}$ is constant (say \pm 1 ) when all $\left|x_{i}-y_{j}\right|>2 x_{0}$, except for sign changes as each $x_{i}-y_{j}$ varies from $-x_{0}$ to $x_{0}$ in accordance with the internal wave function $\pm \sin \left[\kappa\left(x_{i}-y_{j}\right)\right]$. The condition $\kappa=\sqrt{2 \mu V_{0} / \hbar^{2}}=\pi / 2 x_{0}$ determines the well depth $V_{0}$ such that the scattering length $a_{A B}$ is $-\infty$, and the FTG limit is obtained by letting $x_{0} \rightarrow 0$ and $V_{0} \rightarrow \infty$ in accordance with this condition. In this limit the internal wave function becomes invisible and $\Psi_{0}$ appears to jump discontinuously between \pm 1 whenever an A-particle passes a B-particle, but there are hidden nodes at $x_{i}-y_{j}=0$ at the centers of the wells. The ground state energy $E_{0}=0$, generalizing the situation for the pure FTG gas [2, 8, 9, 12]. $\Psi_{0}$ maps to a "model state" $\Psi_{M 0}$ consisting of two noninteracting ideal Bose gases totally Bose-Einstein condensed into their ground orbital, which is a trivial constant for periodic boundary conditions: $\Psi_{0}=\Psi_{M 0} M=M$ and $\Psi_{M 0}=1$ where $M$ is the mapping function

$$
M\left(x_{1}, \cdots, x_{N_{A}} ; y_{1}, \cdots, y_{N_{B}}\right)=\prod_{i=1}^{N_{A}} \prod_{j=1}^{N_{B}} \operatorname{sgn}\left(x_{i}-y_{j}\right)
$$

where the sign function $\operatorname{sgn}(x)$ is $+1(-1)$ if $x>0(x<$ $0)$. Although the mapped bosonic state $\Psi_{M 0}$ is a trivial constant outside the square wells, the interior wave function $\pm \sin \left(\kappa\left|x_{i}-y_{j}\right|\right)$ vanishes with cusps at $x_{i}-y_{j}=0$. Therefore, physical consistency requires the presence of a zero-diameter hard core interaction added to the square well. The mapped Bose gas is then not truly ideal, but rather a TG gas with superimposed attractive well, whose nontrivial interior wave function becomes invisible in the zero-range limit, simulating a mixture of two noninteracting ideal Bose gases insofar as the energy and exterior wave function are concerned. The densities of components $\mathrm{A}$ and $\mathrm{B}$ are trivial constants in the ground state, but the off-diagonal elements of the reduced density matrices of $\Psi_{0}$ are quite nontrivial and interesting, due to the effects of the discontinuities in $M$; they will be discussed in later sections.

The periodic boundary conditions impose constraints on the values of $N_{A}$ and $N_{B}$. Suppose that the positions of all B-bosons and all but one A-boson, say $x_{1}$, are fixed on the open interval $(0, \mathrm{~L})$. Starting with that A-boson at $x_{1}>0$ but to the left of all the other particles and moving it to a position $x_{1}<L$ but to the right of all the others, one counts $N_{B}$ sign changes of $\Psi_{0}$ due to the AB contact nodes, so $N_{B}$ must be even for $L$-periodicity in the A-boson coordinates. Repeating this process with all A-bosons and all but one B-boson fixed and moving that B-boson instead, one counts $N_{A}$ sign changes; hence $N_{A}$ must be even for $L$-periodicity in the B-boson coordinates.

One-particle density matrices and momentum distributions: Generalizing the derivation in 12, 16, 17], one finds that the one-particle density matrix of component $\mathrm{A}$ is

$$
\rho_{1 A}\left(x, x^{\prime}\right)=N_{A} L^{-N_{A}-N_{B}} \int \Psi_{0}\left(x, x_{2}, \cdots, x_{N_{A}} ; Y\right) \Psi_{0}\left(x^{\prime}, x_{2}, \cdots, x_{N_{A}} ; Y\right) d x_{2} \cdots d x_{N_{A}} d Y=n_{A}\left[I\left(x, x^{\prime}\right)\right]^{N_{B}}
$$


where $Y=\left(y_{1}, \cdots, y_{N_{B}}\right), n_{A}=N_{A} / L$, and $I\left(x, x^{\prime}\right)=$ $L^{-1} \int_{-L / 2}^{L / 2} \operatorname{sgn}(x-\xi) \operatorname{sgn}\left(x^{\prime}-\xi\right) d \xi=1-2\left|x-x^{\prime}\right| / L$. In the thermodynamic limit where $N_{B} \rightarrow \infty$ and $L \rightarrow \infty$ such that $N_{B} / L \rightarrow n_{B}$ with finite and nonzero number density $n_{F} n_{B}$, one has $\left[I\left(x, x^{\prime}\right)\right]^{N_{B}} \rightarrow e^{-2 n_{B}\left|x-x^{\prime}\right|}$ in analogy with the one-component FTG gas case 12, 16, 17]. Hence $\rho_{1 A}\left(x, x^{\prime}\right)=n_{A} e^{-2 n_{B}\left|x-x^{\prime}\right|}$, and by interchange of $\mathrm{A}$ and $\mathrm{B} \rho_{1 B}\left(y, y^{\prime}\right)=n_{B} e^{-2 n_{A}\left|x-x^{\prime}\right|}$. Their Fourier transforms $n_{k A}$ and $n_{k B}$, normalized to $\sum_{k} n_{k A}=N_{A}$ and $\sum_{k} n_{k B}=N_{B}$ where the allowed momenta are $k=\nu 2 \pi / L$ with $\nu=0, \pm 1, \pm 2, \cdots)$, are Lorentzian discrete momentum distributions $n_{k A}=\frac{4 n_{A} n_{B}}{4 n_{B}^{2}+k^{2}}$ and $n_{k B}=\frac{4 n_{A} n_{B}}{4 n_{A}^{2}+k^{2}}$. The Fermi-like Lorentzian shapes are strong modifications of the ideal Bose gas distributions $N_{A} \delta k 0$ and $N_{B} \delta k 0$ due to the infinite $\mathrm{AB}$ attraction; no trace of BEC of components $\mathrm{A}$ and $\mathrm{B}$, and of the associated ODLRO of $\rho_{1 A}$ and $\rho_{1 B}$, remains. Nevertheless, at $k=0 n_{k A}$ reduces to $n_{A} / n_{B}$ which increases without limit as the B-component density $n_{B}$ falls to zero for fixed $n_{A}$, and in fact the continuous momentum distribution $(L / 2 \pi) n_{k A}$ reduces to a representation of the ideal Bose gas distribution $N_{A} \delta(k)$ as $n_{B} \rightarrow 0 . n_{k B}$ has the same behavior, with A and B interchanged.

Two-particle density matrices, pairing, and ODLRO: The two-particle A-component and B-component density matrices $\rho_{2 A A}\left(x_{1}, x_{2} ; x_{1}^{\prime}, x_{2}^{\prime}\right)$ and $\rho_{2 B B}\left(y_{1}, y_{2} ; y_{1}^{\prime}, y_{2}^{\prime}\right)$ can also be evaluated in closed form by generalization of (3) and the derivation for the one-component FTG gas in [12]. For component A

$$
\begin{aligned}
& \rho_{2 A A}\left(x_{1}, x_{2} ; x_{1}^{\prime}, x_{2}^{\prime}\right)=N_{A}\left(N_{A}-1\right) L^{-\left(N_{A}+N_{B}\right)} \\
\times & \int \Psi_{0}\left(x_{1}, x_{2}, x_{3}, \cdots, x_{N_{A}} ; Y\right) \\
\times & \Psi_{0}\left(x_{1}^{\prime}, x_{2}^{\prime}, x_{3}, \cdots, x_{N_{A}} ; Y\right) d x_{3} \cdots d x_{N_{A}} d Y
\end{aligned}
$$

Using $\Psi_{0}\left(x_{1}, x_{2}, x_{3}, \cdots, x_{N_{A}} ; Y\right)=\prod_{j=1}^{N_{B}} \operatorname{sgn}\left(x_{1}-\right.$ $\left.y_{j}\right) \operatorname{sgn}\left(x_{2}-y_{j}\right)$ one finds in the thermodynamic limit $\rho_{2 A A}\left(x_{1}, x_{2} ; x_{1}^{\prime}, x_{2}^{\prime}\right)=n_{A}^{2} e^{2 n_{B}\left(z_{1}-z_{2}+z_{3}-z_{4}\right)}$ where $z_{1} \leq$ $z_{2} \leq z_{3} \leq z_{4}$ are the arguments $\left(x_{1}, x_{2} ; x_{1}^{\prime}, x_{2}^{\prime}\right)$ in ascending order. If $x_{1}<x_{2}<x_{1}^{\prime}<x_{2}^{\prime}$ then $\rho_{2 A A}=n_{A}^{2} e^{-2 n_{B}\left|x_{1}-x_{2}\right|} e^{-2 n_{B}\left|x_{1}^{\prime}-x_{2}^{\prime}\right|}$. Generalizing the argument in [12] one sees that if the variable pairs $\left(x_{1}, x_{2}\right)$ and $\left(x_{1}^{\prime}, x_{2}^{\prime}\right)$ are separated to arbitrary distance while keeping $\left|x_{1}-x_{2}\right|$ and $\left|x_{1}^{\prime}-x_{2}^{\prime}\right|$ fixed, then $\rho_{2 A A}$ remains constant, signalling AA-pair ODLRO associated with a leading term $\lambda_{1 A A} u_{1 A}\left(x_{1}, x_{2}\right) u_{1 A}\left(x_{1}^{\prime}, x_{2}^{\prime}\right)$ in the spectral representation of $\rho_{2 A A}$, with eigenfunction $u_{1 A}\left(x_{1}, x_{2}\right)=\mathcal{C}_{A} e^{-2 n_{B}\left|x_{1}-x_{2}\right|}$, normalization constant $\mathcal{C}_{A}=\sqrt{2 n_{B} / L}$, and macroscopic eigenvalue $\lambda_{1 A A}=$ $n_{A}^{2} / \mathcal{C}_{A}^{2}=n_{A} N_{A} / 2 n_{B}$. There is a BEC-BCS crossover from AA-pair BEC when $n_{B} \gg n_{A}$ and the range of $u_{1 A}$ is $\ll 1 / n_{A}$ implying tightly bound AA pairs, to AApair superconductivity when $n_{B} \ll n_{A}$ and the range of $u_{1 A}$ is $\gg 1 / n_{A}$ implying extended and strongly overlapping AA Cooper pairs. Since $\rho_{2 B B}\left(y_{1}, y_{2} ; y_{1}^{\prime}, y_{2}^{\prime}\right)$ exhibits the same behavior with $\mathrm{A}$ and $\mathrm{B}$ interchanged, one concludes that when $n_{B} \gg n_{A}$ there is coexistence of BEC of AA pairs and superconductivity of BB pairs, and when $n_{B} \ll n_{A}$ The AA and BB pairing is a purely off-diagonal phenomenon, both in the case of superconductive ODLRO with weakly bound Cooper pairs and in the case of BEC of tightly-bound pairs. There is no diagonal $\mathrm{AA}, \mathrm{BB}$, or $\mathrm{AB}$ order; the pair distribution functions $D_{A A}\left(x_{1}, x_{2}\right)=n_{A}^{-2} \rho_{2 A A}\left(x_{1}, x_{2} ; x_{1}, x_{2}\right)$, $D_{B B}\left(y_{1}, y_{2}\right)=n_{B}^{-2} \rho_{2 B B}\left(y_{1}, y_{2} ; y_{1}, y_{2}\right)$, and $D_{A B}(x, y)=$ $\left(n_{A} n_{B}\right)^{-1} \rho_{2 A B}(x, y ; x, y)$ are all constant, as is most easily seen by noting that (a) our system of A-bosons and B-bosons with FTG AB attraction maps to a mixture of ideal A-Bose and B-Bose gases with no AB interaction, and (b) diagonal density matrix elements are invariant under mapping via Eq. (2). This generalizes the previous result for the pure FTG gas, where there is superconductive ODLRO but the pair distribution function is constant [12].

The two-particle $\mathrm{AB}$ density matrix is also of interest. By a derivation paralleling that for $\rho_{2 A A}$ and $\rho_{2 B B}$ one finds in the thermodynamic limit

$$
\begin{aligned}
\rho_{2 A B}\left(x, y ; x^{\prime}, y^{\prime}\right) & =n_{A} n_{B} \operatorname{sgn}(x-y) \operatorname{sgn}\left(x^{\prime}-y^{\prime}\right) \\
& \times e^{-2 n_{B}\left|x-x^{\prime}\right|} e^{-2 n_{A}\left|y-y^{\prime}\right|} .
\end{aligned}
$$

Suppose that $x^{\prime}=x+d$ and $y^{\prime}=y+d$. Then $\rho_{2 A B}=n_{A} n_{B} e^{-2\left(n_{A}+n_{B}\right) d}$ which vanishes exponentially as $d \rightarrow \infty$. It follows that there is no AB-pair ODLRO in spite of the strong $\mathrm{AB}$ attraction. It is informative in this connection to compare and contrast two cases (a) two ideal Bose gases $\mathrm{A}$ and $\mathrm{B}$ with no $\mathrm{AB}$ interactions, and (b) the present case, two ideal Bose gases $\mathrm{A}$ and $\mathrm{B}$ with FTG AB interactions. In case (a) there is complete BEC of both components $\mathrm{A}$ and $\mathrm{B}$, the many-body ground state is a trivial constant, $\rho_{2 A A}, \rho_{2 B B}$, and $\rho_{2 A B}$ are also constant, and hence all three of these density matrices exhibit ODLRO. However, the AA and BB ODLRO is a trivial consequence of the trivial ground state structure and more generally, follows from the ODLRO of $\rho_{1 A}$ and $\rho_{1 B}$ [19] and implies no true AA or BB pairing correlation. In the present case (b), there is no BEC of component $\mathrm{A}$ or $\mathrm{B}$ and hence no ODLRO of $\rho_{1 A}$ or $\rho_{1 B}$, but there is ODLRO of both $\rho_{2 A A}$ and $\rho_{2 B B}$, implying both $\mathrm{AA}$ and $\mathrm{BB}$ pairing, since $u_{1 A}$ and $u_{1 B}$ have finite range. On the other hand, $\rho_{2 A B}$ has no ODLRO.

Quantum phase transition: Suppose now that in addition to the odd-wave AB interaction of FTG form, there is also an even-wave $\mathrm{AB}$ interaction of LL delta function form [15], $v_{e}\left(x_{i}-y_{j}\right)=g_{A B} \delta\left(x_{i}-y_{j}\right)$ with $g_{A B}>0$ (repulsive interaction). Recall that before passing to the infinitely narrow well limit $x_{0} \rightarrow 0, V_{0} \rightarrow \infty$ of the FTG interaction, the ground state in the case of no even-wave interaction has nodes at $\mathrm{AB}$ contact due to the internal wave function $\sin \kappa\left(x_{i}-y_{j}\right)$. These nodes kill the even-wave interaction, so that the ground state in the presence of the even-wave interaction is the same as that 
in the absence of an even-wave interaction, which has energy zero. Any state without AB contact nodes has positive energy in the presence of the even-wave repulsion, since then the odd-wave FTG interaction is killed; the system develops $\mathrm{AB}$ contact nodes spontaneously in the presence of the FTG odd-wave attraction in order to lower its energy by killing the even-wave repulsion. Now suppose that there is no even-wave interaction, but the odd-wave attraction is weakened by carrying out the limit $x_{0} \rightarrow 0$ and $V_{0} \rightarrow \infty$ in such a way as to produce a large but finite negative odd-wave scattering length, $-\infty<a_{A B o}<0$. So far the FTG limit where $\kappa x_{0}=\frac{\pi}{2}$ and $a_{A B o}=-\infty$ has been assumed, but more generally if $a_{A B o}$ is negative and finite, then $\kappa x_{0}=\frac{\pi}{2}-\frac{2 x_{0}}{\pi\left|a_{A B o}\right|}$ as $x_{0} \rightarrow 0[9,18]$. The ground state $\Psi_{0}$ in that case maps via (2) to a model ground state $\Psi_{M 0}$ consisting of two Bose gases $\mathrm{A}$ and $\mathrm{B}$ with no $\mathrm{AA}$ or $\mathrm{BB}$ interaction but an even-wave $\mathrm{AB}$ interaction of LL form $g_{A B}^{\prime} \delta\left(x_{i}-y_{j}\right)$ with $g_{A B}^{\prime}=\hbar^{2} / \mu\left|a_{A B o}\right|$ and positive energy. If one generalizes further by adding a nonzero even-wave interaction $g_{A B} \delta\left(x_{i}-y_{j}\right)$ again, then so long as the ground state $\Psi_{0}$ is retained unchanged with $\mathrm{AB}$ contact nodes, the evenwave interaction will be killed and will have no effect. However, if $g_{A B}<g_{A B}^{\prime}=\hbar^{2} / \mu\left|a_{A B o}\right|$, then a ground state with no $\mathrm{AB}$ contact nodes has lower energy, since then it is the odd-wave interaction which is killed, instead of the even-wave interaction. It follows that there is a quantum phase transition between a phase with no $\mathrm{AB}$ contact nodes, which has lower energy when $g_{A B}<g_{A B}^{\prime}$, and a phase with $\mathrm{AB}$ contact nodes, which has lower energy when $g_{A B}>g_{A B}^{\prime}$. This is very similar to the ferromagnetic-antiferromagnetic phase transition in the 1D spinor Fermi gas with both even and odd-wave interactions [20, 21].

Stronger $A B$ attraction: It was pointed out above that if $-\infty<a_{A B o}<0$ then $\kappa x_{0}=\frac{\pi}{2}-\frac{2 x_{0}}{\pi\left|a_{A B o}\right|}$ as $x_{0} \rightarrow 0$, or without the absolute value signs $\kappa x_{0}=\frac{\pi}{2}+\frac{2 x_{0}}{\pi a_{A B o}}$. In analogy with the case of fermions discussed recently [22], for an interaction of FTG form the same relation (without absolute value signs) holds if $a_{A B o}>0$, in which case the model state $\Psi_{M 0}$ generated by the mapping (2) has an attractive LL AB interaction $g_{A B} \delta\left(x_{i}-y_{j}\right)$ with $g_{A B}=-\hbar^{2} / \mu a_{A B o}<0$ and negative energy. The $N=2$ model ground state is bound, $\Psi_{0 M}=e^{-|x-y| / a_{A B o}}$ with energy $E_{0}=-\frac{\hbar^{2}}{2 \mu a_{A B o}^{2}}$, and the corresponding actual physical state is $\Psi_{0}=\operatorname{sgn}(x-y) e^{-|x-y| / a_{A B o}}$, with the same energy. The exact solution for both $N_{A}>2$ and $N_{B}>2$ is not known, but if $\Psi_{M 0}$ has Bijl-Jastrow form $\Psi_{M 0}=\mathfrak{N} \prod_{i=1}^{N_{A}} \prod_{j=1}^{N_{B}} f\left(\left|x_{i}-y_{j}\right|\right)$ where $\mathfrak{N}$ is a normalization constant and $f\left(\left|x_{i}-y_{j}\right|\right) \approx e^{-\left|x_{i}-y_{j}\right| / a_{A B} \text { for }}$ $\left|x_{i}-y_{j}\right| \ll\left(\sqrt{n_{A} n_{B}}\right)^{-1}$, then in the neighborhood of each $x_{i}-y_{j}=0$ the wave function reduces to that of an AB dimer. The behavior of $\Psi_{M 0}$ as $x_{i}$ recedes to distances $x_{i} \gg\left(\sqrt{n_{A} n_{B}}\right)^{-1}$ from $y_{j}$ will be controlled by proximity of $x_{i}$ to other B-particles, not $y_{j}$. If $f(\xi) \rightarrow \lambda$ for $\xi \gg\left(\sqrt{n_{A} n_{B}}\right)^{-1}$ where $\lambda$ is some nonzero constant, then $\mathfrak{N}=\lambda^{-N_{B}}$. The physical state $\Psi_{0}$ generated by the mapping (2) then has reduced density matrices reducible to $1 \mathrm{D}$ integrals as previously. However, in the thermodynamic limit $\rho_{2 A B}$ reduces to the previous expression (5), with no AB-pair ODLRO. A better approximation to $\Psi_{0}$ might reverse this conclusion, so this model deserves further study.

Outlook: In view of the surprising properties of this model, experimental study of mixtures of weakly interacting A-bosons and B-bosons in tight waveguides with strong 1D AB attractions [2] induced by a p-wave AB Feshbach resonance [10] should be fruitful. In particular, one could look for the predicted quantum phase transition.

I thank Peter Reynolds and Gregory Astrakharchik for helpful comments on drafts of this work.

* Electronic address: girardeau@optics.arizona.edu

[1] M. Olshanii, Phys. Rev. Lett. 81, 938 (1998).

[2] B.E. Granger and D. Blume, Phys. Rev. Lett. 92, 133202 (2004).

[3] J.L. Roberts et al., Phys. Rev. Lett. 86, 4211 (2001).

[4] B. Paredes, et al., Nature 429, 277 (2004); T. Kinoshita, T.R. Wenger, and D.S. Weiss, Science 305, 1125 (2004).

[5] T. Kinoshita, T.R. Wenger, and D.S. Weiss, Phys. Rev. Lett. 95, 190406 (2005).

[6] T. Kinoshita, T.R. Wenger, and D.S. Weiss, Nature 440, 900 (2006).

[7] M. Girardeau, J. Math. Phys. 1, 516 (1960); M.D. Girardeau, Phys. Rev. 139, B500 (1965), Secs. 2, 3, and 6.

[8] M.D. Girardeau and M. Olshanii, Phys. Rev. A 70, 023608 (2004).

[9] M.D. Girardeau, Hieu Nguyen, and M. Olshanii, Optics Communications 243, 3 (2004).

[10] C. Ticknor, C.A. Regal, D.S. Jin, and J.L. Bohn, Phys. Rev. A 69, 042712 (2004).

[11] T. Cheon and T. Shigehara, Phys. Lett. A 243, 111 (1998) and Phys. Rev. Lett. 82, 2536 (1999).

[12] M.D. Girardeau and A. Minguzzi, Phys. Rev. Lett. 96, 080404 (2006).

[13] M.D. Girardeau and A. Minguzzi, Phys. Rev. Lett. 99, 230402 (2007).

[14] B.Y. Fang, P. Vignolo,C. Miniatura, and A. Minguzzi, arXiv:0809.4419

[15] E.H. Lieb and W. Liniger, Phys. Rev. 130, 1605 (1963).

[16] M.D. Girardeau and E.M. Wright, Phys. Rev. Lett. 95, 010406 (2005).

[17] S.A. Bender, K.D. Erker, and B.E. Granger, Phys. Rev. Lett. 95, 230404 (2005).

[18] M.D. Girardeau and M. Olshanii, arXiv:cond-mat/0309396

[19] C.N. Yang, Rev. Mod. Phys. 34, 694 (1962).

[20] See pp. 19-20 of [9].

[21] M.D. Girardeau, Phys. Rev. Lett. 97, 210401 (2006).

[22] M.D. Girardeau and E.M. Wright, Phys. Rev. A 77, 043612 (2008). 